Más allá de la torre de marfil: un modelo para potenciar las comunidades de aprendizaje informal y desarrollo mediante prácticas educativas abiertas

\title{
Tony Coughlan
}

t.coughlan@open.ac.uk

The Open University, Reino Unido

\section{Leigh-Anne Perryman}

leigh.a.perryman@open.ac.uk

Institute of Educational Technology, The Open University, Reino Unido

\section{Cita recomendada}

COUGHLAN, Tony; PERRYMAN, Leigh-Anne (2013). «Más allá de la torre de marfil: un modelo para potenciar las comunidades de aprendizaje informal y desarrollo mediante prácticas educativas abiertas». En: «La informalización de la educación» [monográfico en línea]. Revista de Universidad y Sociedad del Conocimiento (RUSC). Vol. 10, n. 1 , págs. 135-150. UOC. [Fecha de consulta: dd/mm/aa]. $<$ http://rusc.uoc.edu/ojs/index.php/rusc/article/view/v10n1-coughlan-perryman/v10n1-coughlanperryman-es>

$<$ http://dx.doi.org/10.7238/rusc.v10i1.1586>

ISSN 1698-580X 


\title{
Resumen
}

Los recursos educativos abiertos (REA) y las prácticas educativas abiertas (PEA) tienen un impacto cada vez mayor en el aprendizaje para adultos, ya que proporcionan educación gratuita de alta calidad a un creciente número de personas. Sin embargo, la distribución vertical que caracteriza a los cursos universitarios disponibles en la actual oferta educativa no se adecua necesariamente a contextos como el desarrollo profesional continuo (DPC). Este artículo sostiene que un nuevo planteamiento que permita pasar de un enfoque centrado en el proveedor a otro que gravite alrededor de la necesidad, basado en las teorías del aprendizaje informal, podría incrementar el impacto positivo de los REA y las PEA más allá de la torre de marfil en qué está hoy situada la educación superior.

Para explorar este planteamiento, nos hemos centrado en los requisitos de una comunidad específica que no pertenece al ámbito de la educación superior -formadores del sector voluntario del Reino Unido- para diseñar un modelo que pueda aplicarse de forma amplia a una comunidad de aprendizaje sostenible y en línea centrada en REA y PEA. El modelo se basa en una encuesta reciente realizada a formadores voluntarios, que establecía su necesidad de disponer de recursos gratuitos de alta calidad y su deseo de entablar relaciones más productivas con sus colegas, así como en un informe sobre comunidades en línea pertenecientes o no al sector voluntario.

El modelo que proponemos presta la misma atención a los recursos de aprendizaje que a la sociabilidad del grupo. Para ello, los investigadores y los profesionales trabajan conjuntamente para adaptar y crear materiales de aprendizaje y poner en común conocimientos y experiencias a través de foros de discusión y otras actividades en colaboración. El modelo plantea una dimensión explícita de mejora de competencias basada en la teoría de las comunidades de práctica (CP) y un sistema de gestión de la reputación que incentiva la participación. Se trata de un modelo único para la creación de una comunidad pan-organizativa totalmente abierta en cuanto a recursos y posibilidad de afiliación. Si bien el modelo que se presenta en este artículo se centra básicamente en el sector voluntario, también podría aplicarse de forma más amplia a otros sectores, lo que permitiría que las comunidades de práctica se beneficiaran de recursos diseñados a medida y de contribuciones académicas, y que las universidades participantes vieran que sus REA se utilizan y reutilizan de forma más amplia para el DPC a través del aprendizaje informal.

\section{Palabras clave}

prácticas educativas abiertas, aprendizaje informal, recursos educativos abiertos, desarrollo profesional continuo, comunidades de práctica, gestión de la reputación

\section{Beyond the Ivory Tower: A Model for Nurturing Informal Learning and Development Communities through Open Educational Practices}

\begin{abstract}
Open Educational Resources (OER) and Open Educational Practices (OEP) are making an ever-growing impact on the field of adult learning, offering free high-quality education to increasing numbers of people. However, the top-down distribution of weighty university courses that typifies current provision is not necessarily suitable for contexts such as Continued Professional Development (CPD). This article proposes that a change of focus from a supplier-driven to a needs-led approach, grounded in theories of informal learning, could increase the positive impact of OER and OEP beyond the ivory towers of higher education.

To explore this approach, we focused on the requirements of a specific community outside higher education - trainers in the UK's voluntary sector - in order to design a more broadly applicable model for a sustainable online learning community focused around OER and OEP. The model was informed by a recent survey of voluntary sector trainers establishing their need for high-quality free resources and their desire to
\end{abstract}


develop more productive relationships with their peers, and by evaluation of successful online communities within and outside the voluntary sector.

Ourproposed model gives equal attention to learning resources and group sociality. In it, academics and practitioners work together to adapt and create learning materials and to share each other's knowledge and experiences through discussion forums and other collaborative activities. The model features an explicit up-skilling dimension based on Communities of Practice ( $\mathrm{CO} P$ ) theory and a system of reputation management to incentivise participation. The model is unique in building a pan-organisation community that is entirely open in terms of membership and resources. While the model offered in this article is focused on the voluntary sector, it could also be applied more widely, allowing practitioner communities the benefits of tailored resources and academic input, and collaborating universities the benefit of having their OER used and reused more widely for CPD through informal learning.

\section{Keywords}

open educational practices, informal learning, open educational resources, continuing professional development, communities of practice, reputation management

\section{Introducción: antecedentes y justificación}

Los recursos educativos abiertos (REA) son materiales docentes y de aprendizaje que pueden utilizarse, reutilizarse y a menudo editarse gratuitamente, y que comprenden desde cursos completos a conferencias, imágenes y videos sueltos. Hoy en día, se considera que el movimiento de REA ejerce una importante influencia en la educación a nivel mundial. Por ejemplo, en la última década, el Instituto de Tecnología de Massachusetts (MIT) ha publicado prácticamente todos sus cursos en línea de forma gratuita y, en abril de 2012, anunció su asociación sin ánimo de lucro con la Universidad de Harvard -edX (www.edxonline.org) - para ofrecer cursos en línea de ambas universidades a más de un millón de personas de todo el mundo. En el Reino Unido, la Open University ha puesto a disposición del público más de 600 cursos gratuitos en línea a través de su repositorio OpenLearn (http:// openlearn.open.ac.uk/) y se puede acceder gratuitamente a cientos de materiales de aprendizaje en vídeo y audio en servicios como YouTube EDU e iTunes $U$.

Los beneficios de los REA para alumnos, profesores y centros educativos están claros. Por ejemplo, ayudan a incrementar la participación en la educación poniendo a disposición del público materiales de aprendizaje de alta calidad sin coste alguno para el usuario (Geser, 2007, pág. 21), independientemente de su ubicación geográfica, estatus económico y nivel educativo. Además, brindan a los educadores la posibilidad de ampliar sus estrategias docentes y de aprendizaje, así como sus contenidos temáticos, mientras que las instituciones e individuos que los crean y publican pueden beneficiarse de «un mayor estatus y visibilidad, y una mayor demanda de servicios y productos» (Schmidt, 2007).

El movimiento en defensa de los REA cristalizó hace más de una década a raíz de la creencia en la idea de «apertura»: la noción de que «el conocimiento debería diseminarse y compartirse de forma gratuita a través de internet a beneficio de la sociedad en su conjunto» (Yuan et al., 2007, pág. 1), con las menores restricciones técnicas, legales o de precio posibles. Aunque el movimiento ha hecho lo 
posible por minimizar estas restricciones, el potencial de los REA sigue estando limitado por la tendencia de las instituciones a publicar recursos siguiendo un modelo vertical orientado al proveedor en vez de tener en cuenta las necesidades del usuario final. Guthrie et al. (2008, pág. 20) confirman que «entender las necesidades del usuario es un elemento de primordial importancia, que a menudo queda desatendido» en el movimiento de REA.

Esta carencia se está empezando a contrarrestar gracias a un cambio de énfasis en la puesta en circulación de REA en las prácticas educativas abiertas (PEA) (Cape Town Open Education Declaration, 2008; Guthrie et al., 2008). Ehlers (2011, pág. 4) explica que las PEA son «prácticas que fomentan la (re)utilización y producción de REA a través de políticas institucionales, promueven modelos pedagógicos innovadores y respetan y capacitan a los alumnos como coproductores». Sin embargo el movimiento de REA se localiza mayoritariamente en las universidades, muchas veces exclusivas (Walsh, 2011), lo que sigue siendo una barrera para las personas que no tienen ninguna relación con la educación superior. En efecto, las investigaciones realizadas tanto sobre REA como sobre PEA tienden a centrarse en los estudiantes universitarios, «lo que deja grandes lagunas en nuestra forma de entender su uso por parte del alumno» (Bacsich et al., 2011, págs. 8-9).

Seely Brown y Adler (2008) sugieren que, fuera de la educación superior, los REA funcionan mejor en una comunidad de aprendizaje. Distinguen entre una modalidad de aprendizaje «impulsada por la oferta», en la que los estudiantes crean un «inventario de conocimientos», y una modalidad «impulsada por la demanda» en qué «se privilegia la participación» (Seely Brown y Adler, 2008, pág. 30), argumentando que la modalidad impulsada por la demanda debería sustituir a la impulsada por la oferta para satisfacer los requisitos cambiantes del ámbito laboral y permitir al usuario el aprendizaje a lo largo de la vida, incluso cuando las materias que le interesen sean muy concretas.

Varios autores (p. ej. Weller, 2011) han identificado el potencial de los REA y las PEA en el contexto del desarrollo profesional continuo (DPC) y JISC (2012) ha considerado el potencial de los primeros para extenderse más allá de la educación superior e integrarse en «comunidades de práctica (como materia o disciplina profesional, sectorial, regional)» incluyendo «su uso en el aprendizaje informal u ocupacional», añadiendo que «el puesto de trabajo y la implicación del empresario tienen una gran importancia y merecen una mayor consideración». Sin embargo, hasta el momento poco se ha escrito sobre su funcionamiento.

Este artículo describe los hallazgos de un estudio que explora el potencial de los REA y las PEA para ser usados en una modalidad impulsada por la demanda en el contexto del DPC, en qué el profesorado que participa en la producción de REA colabora con una comunidad existente fuera de la educación superior, aportando soluciones para obtener, crear y adaptar recursos de DPC. Se esboza un modelo para que esta colaboración pueda llevarse a la práctica.

\section{El contexto de la DPC: formadores del sector voluntario del Reino Unido}

A los efectos de este estudio, nos hemos centrado en una comunidad existente que anticipamos que podría beneficiarse en gran medida de los REA y las PEA: formadores del sector voluntario del Reino 
Unido.' Venter y Sung (2009, pág. 47), refiriéndose al desarrollo de los trabajadores de este sector, señalan que «todavía hay barreras significativas para la formación», como la falta de tiempo, el coste y la falta de disponibilidad de una formación adecuada, y observan que «los recursos para la formación de voluntarios son particularmente escasos» (pág. 45).

Dado que nos interesaba explorar las PEA impulsadas por la demanda, era importante establecer primero las necesidades de la comunidad objetivo. Por ello, se realizó una encuesta en línea a 101 instructores voluntarios y responsables de formación del sector ${ }^{2}$ a fin de poder identificar sus prácticas con relación al uso de recursos de aprendizaje y sus conocimientos y actitudes respecto a los REA. Los resultados de la encuesta revelaron la necesidad del sector de disponer de recursos de aprendizaje pertinentes, fiables y de alta calidad, junto con el deseo expresado por todos de colaborar con sus compañeros en ámbitos relacionados con el desarrollo profesional.

Los resultados del estudio indicaron que, por lo general, los formadores no están familiarizados con los REA. Menos de la mitad de los encuestados indicaron conocer OperLearn y YouTube EDU; sólo el 26\% conocía iTunes U y un escaso 16\% había oído hablar del OpenCourseWare del MIT. Únicamente el 4\% afirmó que había usado REA en su formación y un porcentaje incluso menor indicó que había adaptado REA a su contexto formativo específico. Es importante destacar que las respuestas de la encuesta indicaron claramente que los formadores del sector voluntario tendrían que adquirir habilidades adicionales para poder adaptar y desarrollar REA a sus contextos particulares de enseñanza. Por ejemplo, casi todos los instructores indicaron que les resultaba fácil crear y editar documentos en Word y PowerPoint, pero pocos dijeron que sabían editar imágenes, grabaciones de audio y recursos de vídeo/DVD, que suelen formar parte de los REA. Las respuestas de la encuesta pusieron de manifiesto que la formación animaría a usar REA, ya que el 85\% de los instructores indicaron que estarían más dispuestos a emplearlos si recibían esta formación. Uno de los encuestado añadió que los REA ofrecerían «grandes beneficios PERO sólo con una ayuda apropiada, tanto técnica como de contenido».

Al ser preguntados sobre su posible colaboración con la educación superior, los formadores sugirieron que aunque el profesorado universitario podría desempeñar un papel útil para ayudarles a desarrollar una comunidad de práctica (CP), su aportación no debería desarrollarse de forma vertical, de arriba abajo, sino que debería responder a las necesidades de la comunidad y prestar servicios a sus alumnos. En efecto, el $82 \%$ de los encuestados indicaron que unas mejores relaciones con la comunidad académica y con sus propios colegas aumentaría la probabilidad de usar REA. Varios formadores hicieron hincapié en la importancia de ser capaces de adaptarse rápidamente a nuevos retos en respuesta a las necesidades de sus alumnos. Según el comentario de uno de ellos: «Yo doy clases de capacitación en el cumplimiento de normas y es difícil porque la ley cambia a menudo. Me

\footnotetext{
1. En el Reino Unido y en otros países, el sector voluntario recibe varios nombres, como «sector voluntario y comunitario», «tercer sector», «sector sin afán de lucro», y «sector no lucrativo». Las organizaciones también pueden denominarse «benéficas» 0 «ONG».

2. La encuesta se realizó mediante Survey Monkey (www.surveymonkey.com), con una combinación de preguntas abiertas y preguntas cerradas de la escala de Likert. La muestra $(n=410)$ estaba compuesta por instructores y responsables de formación de las listas de correo de tres entidades que aglutinan a varias organizaciones.
} 
parecería muy útil formar parte de una comunidad donde pudiéramos discutir los cambios legislativos y sus implicaciones».

Sólo el 3\% de los encuestados indicaron que el sector voluntario tiene a su alcance suficientes programas gratuitos de buena calidad. Además, muchos de los formadores afirmaron que les resultaba difícil valorar la calidad, el nivel y la vigencia de los recursos formativos existentes: el 57\% señaló que les costaba evaluar la credibilidad de un recurso de aprendizaje; el 54\% que les era difícil juzgar si el contenido estaba actualizado; el $47 \%$ que tenían problemas para evaluar la validez educativa de un recurso; el 45\% que les costaba juzgar su nivel educativo; y el 63\% que tenían dificultades para saber si el contenido se adecuaba a los estándares profesionales nacionales. Por último, al preguntarles si les parecía difícil evaluar la experiencia de sus compañeros, el 75\% de los formadores respondieron que les costaba determinar la capacidad docente y la experiencia de un formador en una materia específica.

\section{Contexto teórico}

Tras establecer las necesidades de los formadores del sector voluntario, recurrimos a varias teorías sobre el aprendizaje informal ${ }^{3}$ a fin de conceptualizar un posible modelo para una comunidad en línea de prácticas abiertas en que los REA y las PEA se usaran a partir de un modelo impulsado por la demanda para apoyar el DPC tanto de voluntarios y empleados como de los instructores responsables de su formación.

\subsection{Aprendizaje informal en línea y DPC}

El aprendizaje informal suele considerarse especialmente apropiado para el desarrollo de la fuerza laboral. Attwell (2007, pág. 4) hace una distinción entre aprendizaje formal e informal al documentar sus investigaciones sobre el uso de e-learning en pequeñas y medianas empresas (PYME). Identifica la popularidad del aprendizaje informal frente al aprendizaje formal para el DPC, explicando que su equipo de investigación «halló una baja adopción de cursos formales» y en cambio detectó «un uso generalizado de internet para el aprendizaje informal a través de búsquedas, grupos en línea, uso de e-mail y tableros de anuncios», en el que Google se destaca como la «aplicación más popular para el aprendizaje». Cross (2007, pág. 192) se hace eco de la observación de Attwell de que gran parte del aprendizaje informal tiene lugar en línea, afirmando que «internet se diseñó para el aprendizaje informal [ ] y está orientado a los usuarios. El usuario tiene la posibilidad de escoger lo que quiere y tomar una parte o el todo».

\footnotetext{
3. Los términos «aprendizaje informal» y «aprendizaje no formal» se utilizan tanto en el ámbito de la investigación educativa como en otras disciplinas, por lo que explorar la distinción entre ambos está fuera del alcance de este estudio. Véase Burbules (2006) para un análisis de este tema. De acuerdo con Attwell (2007), utilizamos el término «aprendizaje informal» para dar cabida a cualquier tipo de aprendizaje fuera del contexto formal.
} 


\subsection{Aprendizaje informal y CP}

La teoría de CP de Lave y Wenger (1996) ofrece un punto de partida útil para el diseño de nuestro modelo. En términos muy generales, una CP es una red de individuos que tienen un interés común. El aprendizaje dentro de una CP tiende a no ser formal y, en cambio, se produce a través de un proceso en el que los usuarios más experimentados transmiten sus conocimientos y habilidades a sus compañeros, mejorando la experiencia compartida de los participantes. Siemens (2006, pág. 40) explica que «el aprendizaje basado en una comunidad» se fundamenta por lo general en «la sabiduría de la masa», generando una «visión polifacética del espacio o de la disciplina» a través del «diálogo social» y la «diversidad de perspectivas». Seely-Brown y Adler (2008, pág. 30) sugieren que «las comunidades de aprendizaje (algunas veces virtuales) que se desarrollan alrededor de una práctica» son la mejor forma de prestar modalidades de aprendizaje impulsadas por la demanda, y añaden que «muchas veces el aprendizaje resultante es informal en vez de producirse en un entorno formalmente estructurado [] y puede ser asistido tanto por una presencia física como virtual y por la colaboración entre los neófitos y los profesionales/investigadores» (Seely Brown y Adler, 2008, pág. 30).

\section{Evaluación de las comunidades en línea que llevan a cabo prácticas abiertas}

Downes (2007b, pág. 26) señala que «cuando las redes [de aprendizaje] están bien diseñadas, facilitan el aprendizaje». Por lo tanto, después de establecer las necesidades de los formadores del sector voluntario, se evaluaron dos comunidades en línea, con el objetivo de identificar los factores que contribuían a su éxito y que podríamos incluir en nuestro modelo.

Bouman et al. (2008) sostienen que «determinadas infraestructuras de software social son capaces de activar ciertos mecanismos que promueven la participación en actividades sociales en línea y fuera de línea» o que favorecen la «socialización» (pág. 5). Identifican cuatro ámbitos de sociabilidad que el software social debería abordar para incentivar la participación:

- Construcción de identidad, para ayudar a mejorar la confianza y la conectividad entre los participantes que comparten los mismos objetivos y valores.

- Habilitación de la práctica (tanto social como laboral).

- Autorrealización personal, mediante la cual se facilita que «las personas evolucionen utilizando su entorno social para aprender a descubrir nuevas perspectivas» (pág. 11).

- Imitación de la realidad, de modo que los «usuarios [ ] sean más proclives a utilizar sistemas de software que tengan que ver con sus rutinas diarias, sus prácticas y su lenguaje» (pág. 10).

Para evaluar el diseño de dos comunidades en línea especialmente destacadas por sus altos niveles de participación, se utilizó la teoría de los «desencadenantes de la sociabilidad» de Bouman et al.: 
- MERLOT: La organización sin ánimo de lucro MERLOT, perteneciente al sector de la educación superior (www.merlot.org), dispone de una colección de 35.000 REA, algunos de los cuales están revisados por miembros de la organización y por una comunidad abierta en línea compuesta por profesores y alumnos, que evalúan estos materiales de aprendizaje y examinan la pedagogía asociada a su uso. MERLOT recibe una media de 75.320 visitas al mes y en marzo de 2012 tenía 100.380 miembros registrados en todo el mundo.

- rightsnet: Esta comunidad de asesores de derechos sociales, perteneciente al sector voluntario del Reino Unido (www.rightsnet.org.uk), recibe un promedio de 40.000 visitas al mes y en marzo de 2012 se habían afiliado 7.500 personas y 1.500 organizaciones. Casi todos sus miembros son asesores de derechos sociales, por lo que funciona como una comunidad de «segundo nivel», capaz de beneficiar indirectamente a cientos de miles de clientes. Shawn Mach, director del departamento de servicios sociales de Lasa, una institución sin ánimo de lucro dedicada a los derechos sociales y a la tecnología (www.lasa.org.uk) que administra rightsnet, comenta lo siguiente:

Como organización de segundo nivel, rightsnet tiene un enorme efecto multiplicador por el impacto que ejercen nuestros asesores en sus clientes. Creo que es la manera más efectiva y eficiente de crear una comunidad capacitada para ayudar a los demás. Nosotros simplemente ponemos a disposición de la sociedad a nuestro ejército de asesores para que se ayuden entre síy hagan lo que mejor saben hacer. (Mach, 2012)

Los recursos que utiliza la comunidad rightsnet, entre otros, son noticias actualizadas acerca de los últimos instrumentos legales, resúmenes localizables y con referencias cruzadas sobre la jurisprudencia relevante, 12 foros moderados de discusión que ofrecen ayuda para gestionar casos de clientes, información y calculadoras para conocer el monto de las prestaciones y las últimas noticias sobre ofertas de empleo del sector.

Nuestra evaluación de rightsnet y MERLOT ha sido el punto de partida para el diseño del nuestro propio modelo, que se analiza a continuación.

\section{El modelo: comunidad colaborativa de autoformación basada en prácticas abiertas y aprendizaje informal}

En la figura 1 se ilustra nuestro modelo de comunidad colaborativa de autoformación basada en prácticas abiertas y aprendizaje informal orientada a satisfacer las necesidades de los formadores del sector voluntario.

Cada elemento se analiza en la tabla 1, que presenta las relaciones entre sus componentes, las necesidades expresadas por los formadores del sector voluntario y la teoría que informa nuestro modelo, junto con ejemplos existentes de comunidades que ilustran cada uno de sus elementos.

Mientras conceptualizábamos nuestra propuesta, analizamos numerosas comunidades en línea, tanto para identificar elementos comunitarios de éxito como, más adelante en el proceso de desarro- 


\section{RISE \\ Universities and Knowledge Society Journal}

http://rusc.uoc.edu

Más allá de la torre de marfil...

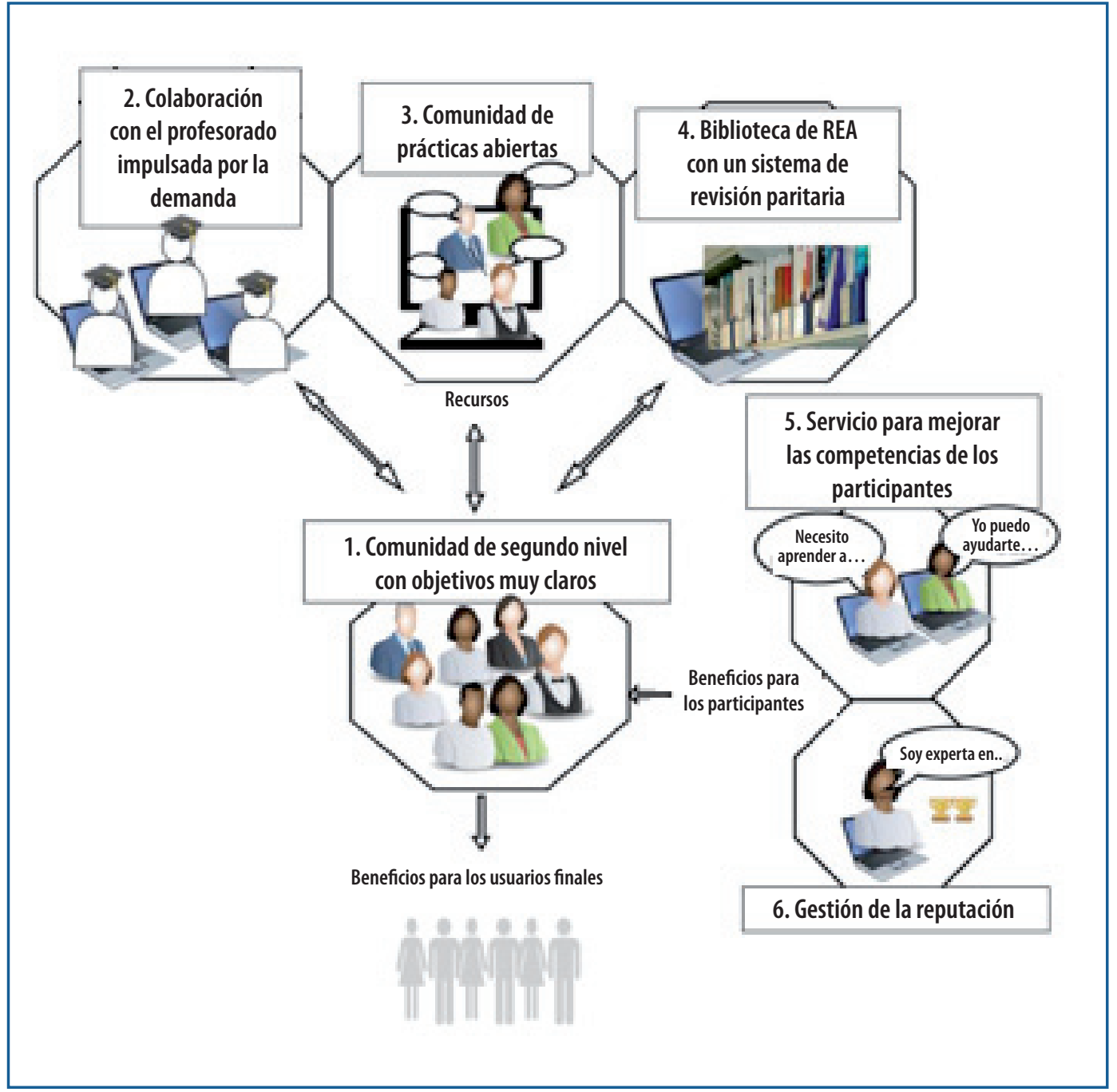

Figura 1. Modelo para una comunidad colaborativa de autoformación basada en prácticas abiertas y aprendizaje informal

Ilo, ver si este modelo ya existía. Algunos elementos del mismo ya se aplican en algunas comunidades en línea como MERLOT y rightsnet, como se indica más arriba. Sin embargo, la particularidad de nuestra propuesta es que sirve para todo tipo de organizaciones, tanto para empleados y voluntarios de distintas organizaciones como para autónomos, y facilita el aprendizaje basándose en la «conectividad», según la cual «el conocimiento se distribuye en una red de conexiones y [ ] el aprendizaje es la capacidad de construir y atravesar estas redes» (Downes, 2007a). Nuestro modelo también es único en el sentido de que no incorpora ningún elemento comercial, ya que se basa en el uso de PEA y REA y no en ofrecer contenido al que solo se pueda tener acceso mediante suscripción.

\subsection{Mantenimiento y sostenibilidad de la comunidad}

Al desarrollar nuestro modelo, también exploramos el mantenimiento y la sostenibilidad de una comunidad de este tipo. Si bien la tecnología utilizada tiene un bajo coste (mantener un sitio web es relativamente barato y puede incluso ser gratuito), los gastos laborales suelen ser mucho más altos. Por 


\begin{tabular}{|c|c|c|c|c|c|}
\hline $\begin{array}{c}\text { Necesidades de los } \\
\text { formadores/responsables } \\
\text { de formación del sector } \\
\text { voluntario }\end{array}$ & & Teoría & & $\begin{array}{c}\text { Solución } \\
\text { (elementos } 1 \text { a } 5 \text { en la figura 1) }\end{array}$ & Ejemplos \\
\hline $\begin{array}{l}\text { Compartir experiencias y } \\
\text { recursos con compañeros } \\
\text { que tienen prioridades } \\
\text { similares. }\end{array}$ & + & $\begin{array}{l}\text { Watts (2010, pág. 192): «El } \\
\text { aprendizaje en línea no es } \\
\text { intrínsecamente social y fun- } \\
\text { ciona mejor con grupos que } \\
\text { ya se conocen entre sí». }\end{array}$ & $=$ & $\begin{array}{l}\text { 1: Comunidad preexistente de } \\
\text { segundo nivel, con objetivos muy } \\
\text { claros y relacionada con un tipo de } \\
\text { trabajo en particular. } \\
\text { Nuestro modelo funciona como una } \\
\text { comunidad de usegundo nivel», } \\
\text { para ayudar a profesionales que } \\
\text { están en primera línea (por ejemplo, } \\
\text { responsables de formación del sector } \\
\text { voluntario) a realizar su trabajo, pero } \\
\text { sin relacionarnos directamente con } \\
\text { los beneficiaros de estos profesionales } \\
\text { (por ejemplo, voluntarios y empleados } \\
\text { del sector voluntario). } \\
\text { Incentivo para la participación: } \\
\text { - Las comunidades en línea con obje- } \\
\text { tivos muy claros y basadas en grupos } \\
\text { naturales tienden a «imitar la reali- } \\
\text { dad» (Bouman et al., 2008) y, como } \\
\text { consecuencia, los miembros de la } \\
\text { comunidad son proclives a disfrutar } \\
\text { de los mismos valores, prácticas y } \\
\text { modalidades de comunicación que } \\
\text { propician el soporte continuado y la } \\
\text { participación en línea. }\end{array}$ & $\begin{array}{l}\text { rightsnet, con objetivos } \\
\text { claramente centrados en un } \\
\text { tipo específico de trabajo, } \\
\text { posee una fuerte identidad } \\
\text { de grupo, manifestada en el } \\
\text { uso de un lenguaje común, } \\
\text { por ejemplo acrónimos, }{ }^{4} \text { la } \\
\text { autoconsciencia de grupo y } \\
\text { referencias directas al grupo } \\
\text { como entidad. } \\
\text { En el foro de rightsnet hay } \\
\text { abundantes recursos, ideas } \\
\text { y experiencias compartidas. } \\
\text { Los usuarios se expresan con } \\
\text { humor, que por lo general } \\
\text { se basa en experiencias y } \\
\text { conocimientos propios del } \\
\text { grupo. }\end{array}$ \\
\hline $\begin{array}{l}\text { Colaborar con profesores } \\
\text { universitarios que respon- } \\
\text { dan a sus necesidades y les } \\
\text { ayuden a desarrollar una CP. }\end{array}$ & + & $\begin{array}{l}\text { Jay Cross (2007, pág. 84), } \\
\text { pionero del e-learning: } \\
\text { Critica la «gestión» vertical, } \\
\text { de arriba abajo, de las } \\
\text { comunidades de aprendizaje } \\
\text { en línea, y sostiene que «una } \\
\text { comunidad no se puede } \\
\text { gobernar. Lo mejor que se } \\
\text { puede hacer es establecer el } \\
\text { contexto, fijar un objetivo y } \\
\text { alimentar el grupo». } \\
\text { Conceptualizando a los } \\
\text { miembros de una comuni- } \\
\text { dad como «alumnos free- } \\
\text { range», Cross (2007, pág. } \\
\text { 223) sugiere que el papel } \\
\text { del profesorado es «prote- } \\
\text { ger su entorno, ofrecerles } \\
\text { elementos que les permitan } \\
\text { evolucionar y dejar que la } \\
\text { naturaleza siga su curso». }\end{array}$ & $=$ & $\begin{array}{l}\text { 2: Colaboración con el profesorado } \\
\text { impulsada por la demanda. } \\
\text { - Los usuarios trabajan con el pro- } \\
\text { fesorado para identificar, crear y } \\
\text { adaptar REA, orientándolos sobre } \\
\text { sus necesidades. } \\
\text { - Inicialmente los profesores pueden } \\
\text { participar como moderadores } \\
\text { en los foros de discusión, pero el } \\
\text { objetivo final es que sean los propios } \\
\text { miembros de la comunidad los que } \\
\text { realicen esta función. } \\
\text { Incentivo para la participación: } \\
\text { - Los participantes tienen autonomía } \\
\text { y una relación con el profesorado } \\
\text { basada en sus propias necesidades. }\end{array}$ & $\begin{array}{l}\text { rightsnet implica colabo- } \\
\text { ración entre especialistas } \\
\text { legales y asesores de dere- } \\
\text { chos sociales que trabajan } \\
\text { en primera línea y suelen } \\
\text { operar según las necesida- } \\
\text { des de la demanda. }\end{array}$ \\
\hline
\end{tabular}

\footnotetext{
4. http://www.rightsnet.org.uk/forums/viewthread/2818/

5. Por ejemplo, http://www.rightsnet.org.uk/forums/viewthread/1661/y

http://www.rightsnet.org.uk/forums/viewthread/1993/
} 


\begin{tabular}{|c|c|c|c|c|c|}
\hline $\begin{array}{c}\text { Necesidades de los } \\
\text { formadores/responsables } \\
\text { de formación del sector } \\
\text { voluntario }\end{array}$ & & Teoría & & $\begin{array}{c}\text { Solución } \\
\text { (elementos } 1 \text { a } 5 \text { en la figura 1) }\end{array}$ & Ejemplos \\
\hline $\begin{array}{l}\text { Colaborar e interactuar } \\
\text { en mayor medida con sus } \\
\text { compañeros. }\end{array}$ & + & $\begin{array}{l}\text { Bouman et al. (2008, pág. } \\
\text { 7): Las comunidades en } \\
\text { línea con éxito combinan la } \\
\text { sociabilidad grupal con la } \\
\text { sociabilidad objetual. } \\
\text { En la sociabilidad grupal, } \\
\text { surge un sentimiento de } \\
\text { pertenencia a una red o una } \\
\text { comunidad (por ejemplo, } \\
\text { Facebook). En la sociabilidad } \\
\text { basada en objetos (por } \\
\text { ejemplo, Wikipedia), «la } \\
\text { experiencia o significado } \\
\text { compartido surge a partir de } \\
\text { los objetos que se considera } \\
\text { que pertenecen a un grupo } \\
\text { determinado o comparten } \\
\text { sus características». }\end{array}$ & $=$ & $\begin{array}{l}\text { 3: Comunidad en línea de prácti- } \\
\text { cas abiertas con foros de discusión } \\
\text { en línea y otras actividades en } \\
\text { colaboración. } \\
\text { Nuestro modelo pretende equilibrar la } \\
\text { sociabilidad grupal con la sociabilidad } \\
\text { objetual, brindando oportunidades } \\
\text { para el aprendizaje informal a través } \\
\text { de la combinación de discusiones } \\
\text { entre compañeros, una biblioteca de } \\
\text { REA y varias actividades realizadas en } \\
\text { colaboración. } \\
\text { Incentivo para la participación: } \\
\text { - Sentido de pertenencia y conecti- } \\
\text { vidad. } \\
\text { - Promoción de la práctica mediante } \\
\text { resolución de problemas en cola- } \\
\text { boración. }\end{array}$ & $\begin{array}{l}\text { rightsnet equilibra la socia- } \\
\text { bilidad grupal y la sociabili- } \\
\text { dad objetual, combinando } \\
\text { los foros de discusión con } \\
\text { una selección limitada pero } \\
\text { abundante de recursos } \\
\text { (aunque muchos de estos } \\
\text { recursos no son abiertos). }\end{array}$ \\
\hline $\begin{array}{l}\text { Tener a su disposición recur- } \\
\text { sos de formación gratuitos } \\
\text { y de gran calidad, así como } \\
\text { información fiable sobre su } \\
\text { calidad, nivel educativo y } \\
\text { vigencia, y conocer su cla- } \\
\text { sificación en los estándares } \\
\text { profesionales nacionales. }\end{array}$ & + & $\begin{array}{l}\text { Bouman et al. (2008): } \\
\text { El hecho de ayudar a los } \\
\text { formadores a evaluar la } \\
\text { calidad de los recursos } \\
\text { cumpliría el desencadenante } \\
\text { de sociabilidad que «facilita } \\
\text { la práctica» de } \\
\text { Bouman et al. }\end{array}$ & $=$ & $\begin{array}{l}\text { 4: Biblioteca de REA equipada con } \\
\text { un sistema de revisión paritaria } \\
\text { para evaluar contenidos. } \\
\text { Los recursos son calificados por los } \\
\text { usuarios a partir de criterios prede- } \\
\text { finidos, con la posibilidad de añadir } \\
\text { comentarios sobre cómo un recurso } \\
\text { puede aplicarse a distintos contextos o } \\
\text { cómo puede mejorarse. } \\
\text { Incentivo para la participación: } \\
\text { - El hecho de revisar los materiales, } \\
\text { añadiendo comentarios y diseñando } \\
\text { ejercicios de aprendizaje ofrece } \\
\text { mayores oportunidades para la } \\
\text { creación de identidad personal y la } \\
\text { autorrealización (dos de los «des- } \\
\text { encadenantes de sociabilidad» de } \\
\text { Bouman). }\end{array}$ & $\begin{array}{l}\text { Los revisores de MERLOT } \\
\text { evalúan materiales de } \\
\text { aprendizaje con criterios que } \\
\text { van desde la calidad de sus } \\
\text { contenidos, su eficacia como } \\
\text { herramienta de enseñanza } \\
\text { y la facilidad de uso para } \\
\text { alumnos y educadores, lo } \\
\text { que da como resultado una } \\
\text { clasificación de } 1 \text { a } 5 \text { estre- } \\
\text { Ilas. Los usuarios también } \\
\text { pueden hacer comentarios } \\
\text { sobre los materiales y ense- } \\
\text { ñar cómo se pueden utilizar } \\
\text { en contextos determinados } \\
\text { o niveles educativos con- } \\
\text { cretos. }\end{array}$ \\
\hline $\begin{array}{l}\text { Disponer de habilidades } \\
\text { adicionales para adaptar } \\
\text { y desarrollar REA con el } \\
\text { objetivo de adecuarlos a su } \\
\text { contexto docente. }\end{array}$ & + & $\begin{array}{l}\text { Lave y Wenger (1996): } \\
\text { Según la teoría de las CP, los } \\
\text { grupos que comparten un } \\
\text { interés o una pasión por algo } \\
\text { aprenden mejor si interac- } \\
\text { túan habitualmente. } \\
\text { Burbules (2006): Según la } \\
\text { teoría de las «comunida- } \\
\text { des de autoformación», } \\
\text { estas están «abiertamente } \\
\text { interesadas en compartir } \\
\text { información, iniciar a los } \\
\text { principiantes y extender su } \\
\text { conocimiento colectivo». }\end{array}$ & $=$ & $\begin{array}{l}\text { 5: Servicio para mejorar las com- } \\
\text { petencias de los participantes a } \\
\text { través de la CP. } \\
\text { Los foros de discusión permiten a los } \\
\text { formadores compartir sus habilidades } \\
\text { y recursos, y trabajar en colaboración } \\
\text { para rediseñar REA existentes ade- } \\
\text { cuándolos a su contexto y crear nuevos } \\
\text { recursos a partir de cero. Inicialmente, } \\
\text { los profesores especialistas en REA } \\
\text { pueden tener un papel de liderazgo } \\
\text { en la mejora de las habilidades de los } \\
\text { formadores, aunque se prevé que la } \\
\text { comunidad se autoformará a través de } \\
\text { un proceso de desarrollo formativo y } \\
\text { habilidades en cascada. } \\
\text { Incentivo para la participación: } \\
\text { - Oportunidades de desarrollo de } \\
\text { formación y habilidades }\end{array}$ & $\begin{array}{l}\text { rightsnet es una comunidad } \\
\text { autoformativa, en la que } \\
\text { los miembros trabajan en } \\
\text { colaboración para compartir } \\
\text { experiencias y solucionar } \\
\text { problemas complejos. } \\
\text { Los revisores de MERLOT se } \\
\text { forman a través de un «Gra- } \\
\text { pe Camp» en línea, en el que } \\
\text { se combinan presentaciones } \\
\text { dirigidas por un instructor } \\
\text { con tareas colaborativas de } \\
\text { revisión. }\end{array}$ \\
\hline
\end{tabular}




\begin{tabular}{|c|c|c|c|c|c|}
\hline $\begin{array}{c}\text { Necesidades de los } \\
\text { formadores/responsables } \\
\text { de formación del sector } \\
\text { voluntario }\end{array}$ & & Teoría & & $\begin{array}{c}\text { Solución } \\
\text { (elementos } 1 \text { a } 5 \text { en la figura 1) }\end{array}$ & Ejemplos \\
\hline $\begin{array}{l}\text { Ser capaces de valorar la } \\
\text { credibilidad y las competen- } \\
\text { cias de sus compañeros. }\end{array}$ & + & $\begin{array}{l}\text { Burbules (2006, pág. 274): } \\
\text { Algunos miembros de las } \\
\text { comunidades en línea «se } \\
\text { distinguen por la frecuencia, } \\
\text { la calidad y la originalidad } \\
\text { de sus aportaciones, así } \\
\text { como por su experiencia o } \\
\text { sus conocimientos recono- } \\
\text { cidos por todos de forma } \\
\text { consensuada». } \\
\text { Los sistemas de gestión de } \\
\text { la reputación ofrecen a los } \\
\text { miembros de una comu- } \\
\text { nidad la oportunidad de } \\
\text { demostrar explíitamente } \\
\text { su valía dentro y fuera de la } \\
\text { comunidad. } \\
\text { Bacon (2012) defiende un } \\
\text { modelo de gestión de la } \\
\text { reputación basado en el «ca- } \\
\text { pital social», y sostiene que } \\
\text { una «comunidad es funda- } \\
\text { mentalmente una economía } \\
\text { social y sus participantes } \\
\text { desarrollan capital social } \\
\text { mediante sus aportaciones» } \\
\text { (pág. 14), según el principio } \\
\text { de la meritrocracia, por la } \\
\text { que cada «miembro tiene } \\
\text { una responsabilidad y un } \\
\text { reconocimiento de acuerdo } \\
\text { con sus logros, méritos y } \\
\text { talento» (pág. 37). }\end{array}$ & $=$ & $\begin{array}{l}\text { 6: Sistema de gestión de la repu- } \\
\text { tación } \\
\text { En el perfil de cada miembro aparece } \\
\text { un distintivo que indica su papel en la } \\
\text { comunidad y cuantifica las tareas que } \\
\text { realiza para la misma, como revisor, } \\
\text { moderador o participante en el foro. } \\
\text { Incentivo para la participación: } \\
\text { - Facilitar la creación de identidad de } \\
\text { los participantes. } \\
\text { - Ofrecer valor para el desarrollo } \\
\text { profesional fuera de la comunidad. } \\
\text { - Permitir que los usuarios valoren la } \\
\text { credibilidad y la experiencia de las } \\
\text { personas que hacen comentarios } \\
\text { sobre los materiales de aprendizaje, } \\
\text { y que por lo tanto «facilitan la } \\
\text { práctica» (Bouman et al., 2008). }\end{array}$ & $\begin{array}{l}\text { MERLOT aplica un sistema } \\
\text { meritocrático de gestión } \\
\text { de la reputación, por el que } \\
\text { despliega automáticamente } \\
\text { un perfil para cada miem- } \\
\text { bro, visible para el público, } \\
\text { además de un resumen } \\
\text { de sus aportaciones a la } \\
\text { comunidad. }\end{array}$ \\
\hline
\end{tabular}

Tabla 1: Componentes de una comunidad autoformativa y colaborativa de prácticas abiertas y aprendizaje informal

eso, nuestro modelo incluye la moderación de las discusiones del foro y el soporte técnico por parte de los miembros de la comunidad. El estudio de caso de rightsnet demuestra que los moderadores pueden surgir entre la comunidad de participantes «veteranos». Sin embargo, por lo general la gente necesita que sus esfuerzos sean recompensados. En rightsnet, el vínculo entre participación comunitaria y beneficio profesional es tan estrecho que los miembros participan voluntariamente para comoderar la comunidad. Sin embargo, lo más probable es que no ocurra lo mismo en comunidades en que la relación entre práctica y participación es menos directa. En esos casos, se prevé que el sistema de gestión de la reputación (elemento 6 del modelo) ayude a incentivar la participación en el funcionamiento de la comunidad, permitiendo que los usuarios exhiban y pongan de manifiesto su credibilidad en el sí de la comunidad y a la vez desarrollen una reputación y una credibilidad fuera de la misma. Mediante la gestión de la reputación, reconociendo y agradeciendo públicamente estas funciones, podrá incentivarse la moderación (y otros papeles necesarios para el desarrollo de una comunidad, como el soporte técnico), junto con la provisión y evaluación de recursos. 


\section{Conclusión}

Nuestro modelo toma los componentes más eficaces de las dos comunidades estudiadas y los combina para satisfacer las necesidades de los formadores del sector voluntario y aprovechar las habilidades y los recursos ofrecidos por los profesores que prestan su colaboración. Aunque se centra en el sector voluntario, podría aplicarse fácilmente a otros sectores, lo que ayudaría a salvar la desconexión entre el aprendizaje informal y el aprendizaje formal que se produce en las instituciones educativas (Attwell, 2007). Futuras investigaciones deberían analizar la manera en que este modelo podría aplicarse a otros sectores, tanto en el Reino Unido como en el resto del mundo, para extender los beneficios de los REA y las PEA a un público más amplio.

Nuestro modelo sigue siendo un proyecto en curso y, como tal, todavía no ha sido puesto a prueba. Además, nuestra intención no es plantear una plataforma de software para el modelo, ya que esto estaría fuera del alcance de este artículo y, puesto que las herramientas en línea evolucionan constantemente, es más importante pensar en la funcionalidad que en el software. Sin embargo, el modelo debería tener la posibilidad de ofrecer un enfoque estructurado pero flexible para ampliar los beneficios de los REA y las PEA más allá de las torres de marfil de la educación superior mediante una comunidad autoformativa de prácticas abiertas basada en el aprendizaje informal y en la colaboración con el profesorado universitario.

\section{Bibliografía}

ATTWELL, G. (2007). «Personal Learning Environments - the future of eLearning?». eLearning Papers. Vol. 2, núm. 1. [Fecha de consulta: 20 de mayo de 2012]. $<$ http://www.elearningeuropa.info/files/media/media11561.pdf>

BACON, J. (2012). The Art of Community: Building the New Age of Participation. Sebastapol, California: O'Reilly Media.

BACSICH, P.; PHILLIPS, B.; BRISTOW, S. F. (2011). Learner Use of Online Educational Resources for Learning (LUREAL) - Final report. [Fecha de consulta: 5 de junio de 2012].

<http://www.jisc.ac.uk/media/documents/programmes/elearning/REA/LUREALfinalreport.docx>

BOUMAN, W.; HOOGENBOOM, T.; JANSEN, R.; SCHOONDORP, M.; DE BRUIN, B.; HUIZING, A. (2008). «The Realm of Sociality: Notes on the Design of Social Software». PrimaVera Working Paper Series. Ámsterdam: Universiteit Van Amsterdam. [Fecha de consulta: 3 de junio de 2012]. $<$ http://primavera.feb.uva.n//PDFdocs/2008-01.pdf>

BURBULES, N. C. (2006). «Self-Educating Communities: Collaboration and Learning through the Internet». En: Z. Bekerman, N. C. Burbules \& D. Silberman-Keller. Learning in Places: The Informal Education Reader. Nueva York: Peter Lang Publishing. Págs. 273-284.

CAPE TOWN OPEN EDUCATION DECLARATION (2008). Cape Town Open Education Declaration. [Fecha de consulta: 20 de julio de 2011]. $<$ www.capetowndeclaration.org/read-the-declaration> 
CROSS, J. (2007). Informal Learning: Rediscovering the Natural Pathways That Inspire Innovation and Performance. San Francisco, California: Pfeiffer/Wiley.

DOWNES, S. (2007a). Half an Hour: What Connectivism Is. [Fecha de consulta: 2 de octubre de 2012]. <http://halfanhour.blogspot.co.uk/2007/02/what-connectivism-is.html>

DOWNES, S. (2007b). «Learning Networks in Practice». Emerging technologies for learning. Vol. 2, págs. 19-27. [Fecha de consulta: 25 de mayo de 2012].

<nparc.cisti-icist.nrc-cnrc.gc.ca/npsi/ctrl?action=rtdoc\&an=8913424\&lang=en>

EHLERS, U.-D. (2011). «Extending the Territory: From Open Educational Resources to Open Educational Practices». Journal of Open, Flexible and Distance Learning. Vol. 15, n 2, págs. 1-10. [Fecha de consulta: 14 de junio de 2012].

<http://journals.akoaotearoa.ac.nz/index.php/JOFDL/article/viewFile/2064/2046>

GESER, G. (2007). Open Educational Practices and Resources - OLCOS Roadmap 2012. [Fecha de consulta: 3 de junio de 2012].

<http://www.olcos.org/cms/upload/docs/olcos_roadmap.pdf>

GUTHRIE, K.; GRIFFITHS, R.; MARON, N. L. (2008). Sustainability and Revenue Models for Online Academic Resources: An Ithaka Report. [Fecha de consulta: 20 de mayo de 2012].

<http://sca.jiscinvolve.org/wp/files/2008/06/sca_ithaka_sustainability_report-final.pdf>

JISC (2012). Open Practice Across Sectors: A briefing paper. [Fecha de consulta: 10 de junio de 2012]. <https://REAsynth.pbworks.com/w/page/49655750/OpenPracticeAcrossSectors>

LANE, A. (2008). «Am I good enough? The mediated use of open educational resources to empower learners in excluded communities». Fifth Pan-Commonwealth Forum on Open Learning, 13-17 de Julio de 2008. Londres, Reino Unido. [Fecha de consulta: 26 de junio de 2012]. <http://oro.open.ac.uk/17829/1/Am_I_good_enough.pdf>

LAVE, J.; WENGER, E. (1996). Situated Learning: Legitimate Peripheral Participation. Cambridge, Reino Unido: Cambridge University Press.

MACH, S. (2012). Interview with Leigh-Anne Perryman, 23 de marzo de 2012.

SCHMIDT, P. (2007). UNESCO REA Toolkit Draft. [Fecha de consulta: 16 de junio de 2012]. <http://wikieducator.org/UNESCO_REA_Toolkit_Draft\#Benefits_of_Open_Educational_Resources> SEELY BROWN, J.; ADLER, R. P. (2008). «Minds on Fire: Open Education, the Long Tail, and Learning 2.0.». EDUCAUSE Review. Vol. 43, n 1, págs. 16-32. [Fecha de consulta: 11 de junio de 2012]. $<$ http://net.educause.edu/ir/library/pdf/ERM0811.pdf>

SIEMENS, G. (2006). Knowing Knowledge. Lulu.com.

VENTER, K.; SUNG, J. (2009). Do Skills Matter? A Literature Review on Skills and Workforce Development in the Third Sector. Londres: Skills Third Sector.

WALSH, T. (2011). Unlocking the Gates: How and why leading universities are opening up access to their courses. Princeton / Oxford: Princeton University Press.

WATTS, N. (2010). «Reflecting On Models For Online Learning In Theory \& Practice». The All Ireland Journal of Teaching and Learning in Higher Education. Vol. 2, no 1, págs. 19.1-19.12. [Fecha de consulta: 13 de junio de 2012]. <http://ojs.aishe.org/index.php/aishe-j/article/viewArticle/2019> 
WELLER, M. (2011). The Digital Scholar: How Technology Is Transforming Scholarly Practice. Basingstoke, Reino Unido: Bloomsbury Academic. $<$ http://dx.doi.org/10.5040/9781849666275>

YUAN, L.; MACNEILL, S.; KRAAN, Wilbert (2007). Open Educational Resources - Opportunities and Challenges for Higher Education. [Fecha de consulta: 2 de junio de 2012]. $<$ http://wiki.cetis.ac.uk/images/0/0b/REA_Briefing_Paper.pdf>

\title{
Sobre los autores
}

\author{
Tony Coughlan \\ t.coughlan@open.ac.uk \\ The Open University, Reino Unido
}

Tony Coughlan es asistente social titulado. Ha realizado un posgrado de Trabajo social y un máster de Educación (formación permanente). Es profesor regional de la Open University, donde lleva la gestión de cursos sobre infancia y juventud y ha colaborado en distintas ocasiones con organizaciones dedicadas a la infancia y la juventud. Además de realizar su trabajo remunerado, es director y miembro del consejo de administración de una organización benéfica dedicada a niños de corta edad.

Es miembro del Centro de Apoyo de Recursos Abiertos en la Educación (Support Centre for Open Resources in Education, www.OpenEd.ac.uk). Entre sus intereses actuales, destaca el uso de recursos educativos abiertos y prácticas educativas abiertas en la infancia. 


\section{RISG \\ Universities and Knowledge Society Journal}

http://rusc.uoc.edu

Más allá de la torre de marfil.

Leigh-Anne Perryman

leigh.a.perryman@open.ac.uk

Institute of Educational Technology, The Open University, Reino Unido

Leigh-Anne Perryman tiene una licenciatura en Humanidades, un máster en Educación inclusiva y un doctorado en Educación artística inclusiva otorgado por The Open University del Reino Unido. Su tesis doctoral exploraba las respuestas cognitivas y afectivas de los adultos frente al arte contemporáneo y sus implicaciones para la pedagogía del arte. Es miembro de la Academia de Educación Superior del Reino Unido y del Instituto para el Aprendizaje. Ha sido profesora de The Open University durante 12 años, donde también ha participado en la creación de materiales docentes y en el diseño de cursos en línea. Entre sus actuales intereses de investigación destacan la inclusión social y educativa y el uso de recursos educativos abiertos y prácticas educativas abiertas dentro del sector voluntario y comunitario. Recientemente ha finalizado un proyecto financiado por el Gobierno británico en el que explora el uso de comunidades de e-learning y soporte mutuo en el sector voluntario.

Institute of Educational Technology

The Open University

Walton Hall

Milton Keynes

MK7 6AA

Reino Unido

www.open.ac.uk

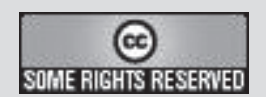

Los textos publicados en esta revista están sujetos -si no se indica lo contrario- a una licencia de Reconocimiento 3.o España de Creative Commons. Puede copiarlos, distribuirlos, comunicarlos públicamente y hacer obras derivadas siempre que reconozca los créditos de las obras (autoría, nombre de la revista, institución editora) de la manera especificada por los autores o por la revista. La licencia completa se puede consultar en http://creativecommons.org/licenses/by/3.0/es/deed.es.

\section{$\bullet \mathrm{UOC}$ \\ Universitat Oberta \\ de Catalunya}

